\title{
Medios de comunicación y política exterior del Estado La prensa y el proceso de paz Ecuador-Perú: 1998 The media and foreign policy The press and the Ecuador-Peru peace process: 1998
}

\begin{abstract}
Sumario
Conforme el nivel de interacción de los actores globales ha avanzado en las últimas décadas, el papel de los medios de comunicación ha aumentado y su papel en la política nacional e internacional se ha hecho más notorio. El impacto que los medios despiertan en la sociedad es una clave para que los ciudadanos opinen y se apropien de los temas que son más cercanos. Este fenómeno ha llamado la atención de varios académicos dispuestos a analizar el rol de los medios como formadores de opinión, como actores políticos y como difusores o exportadores de cultura. ${ }^{1}$ Aquí se analiza un caso muy particular de influencia y participación de la prensa en la política exterior de los Estados y de manera muy precisa en el proceso de paz ecuatorianoperuano de 1998.
\end{abstract}

Juan Francisco Alvarado*

\section{Palabras clave}

Medios de comunicación, prensa, política, política exterior, relaciones internacionales, proceso de paz.

\begin{abstract}
With the level of interaction of global actors progressing during the past decades, the role of the media has increased, and its relevance both in domestic and international politics has become more notorious. The impact the media exert on the society is key whenever citizens express their opinions and embrace issues that are nearest to them. This phenomenon has called the attention of several academics willing to analyze the role of the media as opinion-shapers, political actors, and disseminators and exporters of culture. ${ }^{2}$ The article analyzes a particular case of influence and participation of the press in the foreign policy of States and, more precisely, the Ecuadorian-Peruvian peace process of 1998.
\end{abstract}

\section{Key words}

Media, press, politics, foreign policy, international relations, peace process.

Forma sugerida de citar: $\quad$ ALVARADO, Juan. 2012. "Medios de comunicación y política exterior del Estado. La prensa y el proceso de paz Ecuador-Perú: 1998”, en: Universitas, enero-julio de 2012. Quito: Editorial Abya-Yala, pp. 69-92.

* Sociólogo (PUCE) y Master en Políticas y programas sociales (Universidad de Granada/España).

1 En este tema existen páginas web latinoamericanas dedicadas a criticar el rol de la cultura en nuestras vidas cotidianas, del caso de la norteamericanización y del papel de los medios. Por ejemplo, se puede recurrir a www.razonypalabra.org.mx y revisar en las diferentes publicaciones relacionadas con Comunicación en la Vida Cotidiana, Alcances del periodismo de investigación, industrias de telecomunicación en México con el TLC y Cultura y Comunicación. Así mismo en esta página existen links para otras páginas parecidas en países latinoamericanos.

2 On the subject, there are Latin American websites dedicated to criticizing the roles of culture in our daily lives, "North-Americanization", and the media. For example, one may visit www.razonypalabra.org.mx and review the various publications related to Communication in Daily Life, Scopes of Investigative Journalism, Media Industry in Mexico, the AFTA, and Culture and Communication. Likewise on that page, there are web links to other similar sites in Latin American countries. 
La situación actual de los medios de comunicación ha tenido diferentes repercusiones en la vida cotidiana de los ciudadanos: ha permitido manejar información en tiempo real, levantar la voz individual de los seres humanos, presentar situaciones relevantes al resto de la sociedad y penetrar con fuerza el ámbito internacional. Para lograr la posición en la que se encuentran en la actualidad los medios de comunicación se necesitó de varios adelantos tecnológicos, una mayor adaptación de las sociedades a las tecnologías de la comunicación y una penetración cada vez más eficiente de estas en la vida diaria de las personas.

El rol ya mencionado de los medios les permite involucrarse en otras áreas que anteriormente eran consideradas para otros actores, tal es el caso de la política exterior de un gobierno. Es claro que los actores claves en la política internacional de todo país son el estadista, el diplomático y el militar; en base al planteamiento previo que mencionaba a los medios como formadores de opinión pública, entonces podría existir una conexión con estos actores tradicionales. Así como existen estudios sobre el rol de los medios en relación a su influencia sobre la política interna, recientemente han aparecido estudios también sobre el rol de los medios en la política exterior.

En el caso ecuatoriano en particular, los medios de comunicación lograron articular con el tiempo un gran poder de transmitir información y de mantener altos niveles de aprobación y legitimidad. En cuanto a la formulación de la política exterior, sus actores tradicionales han dejado espacios para la acción de nuevos actores en temas que no han abordado. En la formulación de política exterior es significativo el avance logrado con las explicaciones sobre modelos de toma de decisiones, papel de los negociadores e injerencia de factores externos y nuevos a los mencionados en las teorías clásicas de realismo e idealismo políticos.

Dentro de la política exterior existen los tomadores de decisiones, que generalmente corresponden a diplomáticos y políticos especializados en el tema, pero la injerencia o presión que estos puedan tener por parte de otros actores es importante mencionar (ya que una acción establecida en una agenda de política exterior abarca temas diversos que tienen repercusiones en diferentes ámbitos nacionales e internacionales, de tipo socioeconómico, político y cultural). En términos generales la política exterior de nuestro país se basaba en reacciones al escenario mundial y a las acciones de otros actores. El presente artículo ha sido elaborado a partir de una investigación realizada en 2009 con motivo de una tesis en la cual se enfoca el rol de los medios en la política exterior de 
Ecuador durante 1998 durante la firma del tratado de paz con el Perú. Se pudo identificar insuficiente información sobre el tema, la que pudo ser producida y elaborada durante el trabajo investigativo en base a la siguiente pregunta de investigación:

¿De qué forma han influido los medios de comunicación escritos en la opinión pública, en las acciones de política exterior que el Gobierno ecuatoriano tomó durante la negociación y la firma de la paz con Perú?

La metodología que se planteó se la menciona a continuación:

- Análisis de teorías en base a libros, revistas y publicaciones en internet: esto se realiza en la primera parte de la tesis, ya que es necesario encontrar los enfoques teóricos más apropiados así como los elementos necesarios para poder explicar de manera más detallada el fenómeno a estudiarse.

- Síntesis sobre las bases teóricas que son aplicables en nuestro medio después de confrontaciones realizadas durante el proceso con hechos reales.

- Análisis de editoriales que corresponden a la etapa final de la negociación de paz con Perú: selección de los más relevantes de acuerdo a fechas históricas durante las negociaciones y su clasificación según el contenido. Se ha tomado como base los diarios escritos El Comercio y El Universo como los diarios más importantes a nivel nacional, uno a nivel de la Sierra y el otro a nivel de la Costa. Además se los tomó en cuenta por sus alianzas con diferentes redes de medios de comunicación nacional e internacionales y finalmente por la variedad de opiniones que en sus editoriales se vierten según la importancia de la noticia del día.

- Análisis de boletines de prensa del Ministerio de Relaciones Exteriores (MRREE), que tengan relación con el conflicto: para tener en cuenta la versión oficial del conflicto, que era reproducida por los medios de comunicación.

- Entrevistas a actores claves: las visiones militar, diplomática y académica se contrastan con los hechos y con la forma en que los medios abordaron los eventos que se sucedieron. Para esto se utilizan recortes de editoriales de periódicos en un tiempo determinado, entrevistas con actores vivenciales de la realidad entre 1995 y 1998 y contraste con las teorías de relaciones internacionales ya abordadas. Con todo el análisis realizado, uno puede darse cuenta que la amplitud de los medios de comunicación 
así como el crecimiento de actores ligados a relaciones internacionales permiten mayores estudios.

\section{Enfoque teórico: relación efecto cnn-manufacturing consent}

En las dos corrientes se menciona la importancia de la velocidad de la comunicación así como de la facilidad de tener información las 24 horas del día en el mundo actual; bajo esta premisa es indudable que el avance de la tecnología ha permitido a los medios recopilar información sobre lo que está pasando en el mundo de manera inmediata. El gran problema es la selección de la información por parte de los generadores de noticias y los editores, lo que conlleva manipulaciones por parte de poderes políticos en los medios o viceversa. Los medios pueden dar mayor alcance a un evento particular $u$ omitirlo de la escena pública sin que la gente se dé cuenta. Es por ello que la cobertura juega un papel crucial en el panorama público y en la imagen del evento que se quiere proyectar. Otro problema es que los medios son en su mayoría sostenidos económicamente por auspiciantes y sponsors que también pueden influir en la programación e información que se presenta a la gente.

La teoría del 'efecto CNN' es la más nueva, ha sido ampliamente criticada por sus diferentes interpretaciones. En esencia, propone la capacidad de los medios de delinear agendas políticas y cambiar decisiones políticas. Esta teoría propone además que los medios, especialmente los visuales, han logrado un cambio nunca antes visto en la historia: son los encargados de delinear la atención del público en eventos específicos y, además, proponer cambios en las acciones de diferentes actores nacionales e internacionales. Según los autores que han propuesto esta corriente, el efecto $\mathrm{CNN}$ puede tener tres variantes: el de ser un catalizador de un proceso que se venía gestando, el de ser un freno a cualquier iniciativa política o el de ser el que moldea la agenda que deberá ser tratada.

La teoría de manufacturing consent argumenta que los medios no pueden construir agendas de política exterior, y que más bien los medios pueden ser manipulados como apoyo a políticas gubernamentales; pueden estar en contra o a favor del poder ejecutivo y de ello depende la conformación de gobiernos. Además de que la comunicación se basa en principios comerciales de competitividad, que les obliga a ofrecer al cliente un material que pueda ser vendido sin importar realmente el contenido; pueden actuar también como un arma para 
atacar errores de líderes opositores con el fin de restar su credibilidad. Esta teoría implica una mayor injerencia del poder político en la edición de noticias y en la proyección de opiniones; esto significa que las relaciones son estrechas y pueden llegar al extremo de ofrecer visiones totalmente parcializadas a favor del poder político dominante.

La relación entre medios y formulación de política exterior puede adoptar dos caminos: el primero con actores en política exterior, que puedan tener la capacidad de manejar la atención de los medios hacia ciertos eventos que ellos mismo producen y mantienen, esto tiene que ver con relaciones de poder ocultas y conflicto de intereses. En el otro extremo pueden existir medios, que dependiendo de su cobertura e importancia, pueden dar pautas para el manejo de política exterior y establecer planes de acción a futuro, en este último caso los medios pueden aumentar la atención de la sociedad civil, proponer soluciones, frenar el desarrollo de políticas o crear agendas. Una característica importante de los medios es que logran legitimar la autoridad o la importancia de ciertos actores dentro de los procesos.

Según el autor Michael Brecher, los medios son herramientas para comunicarse con los grupos de poder o para expresar lo que los grupos de poder quieren; sin duda la característica más importante de los medios es que logran legitimar la autoridad o la importancia de ciertos actores frente a la sociedad en general. Es por ello que los medios de comunicación pueden funcionar como promotores de líderes locales que quieran un papel más importante en el exterior así como una mayor difusión de sus ideas y proyectos; especialmente de aquellos sectores políticos que estén sometidos a votación por parte de la población y que, por lo tanto, deben estar siempre informando de sus labores y ser sensibles a la opinión pública. Su impacto será importante dentro del proceso de toma de decisiones, pero no será fundamental para cambiar de manera radical el discurso de los hacedores de política exterior. Como se mencionó anteriormente es más importante para el tomador de decisiones realizar un análisis de riesgos de una posible intervención, la oportunidad de victoria y el apoyo a nivel domestico, antes que saber si un medio en particular hace una cobertura especial o tiene una postura definida sobre el tema.

Los puntos críticos que debe plantear esta relación los enuncia Simon Serfaty, al preguntarse sobre los límites de la «necesidad del pueblo» de saber, de las «responsabilidad del gobierno» de ocultar información critica y del «de- 
recho de los medios» a informar. ${ }^{1}$ En su análisis menciona la competencia de los medios por obtener primicias y la grandiosa red de comunicaciones actual, que no responde a límites estatales y que tampoco logra la "primacía" sobre el control de la información. Más bien, cuando se trata de obtener la noticia más relevante, se puede filtrar la información oficial, con carácter anónimo generalmente; así mismo los medios pueden colaborar con grupos que quieren llamar la atención con sus actos y que no favorecen al status quo de la realidad internacional, como grupos subversivos, separatistas o religiosos que logran notoriedad y reconocimiento mundial para sus proyecciones, las cuales no lograrían sin el efecto mediático de una cobertura en tiempo real.

Para terminar la relación de medios y política exterior, podemos mencionar nuevamente a Serfaty, quien menciona que la prensa tiene un desempeño en el cambio de opinión. ${ }^{2}$ Los cambios que se han producido en los últimos años han abierto las puertas para que los periodistas obtengan mayores informaciones de regímenes cerrados y de fracasos de políticas exteriores. El efecto en la gente es lo que en realidad influye en el cambio de política exterior; los medios cumplen su deber de informar y desde luego deberían instruir sobre temas desconocidos (aunque este papel todavía es discutible sobre los límites de los medios en la educación de la sociedad). La sugerencia que el autor propone es la capacitación de los periodistas, ya que si los cronistas son los "investigadores del presente", entonces deben recibir capacitación adecuada para poder transferir la información hacia el resto del pueblo de manera adecuada y precisa.

Por medio de una capacitación adecuada, los periodistas podrían tener un rol más activo y legítimo dentro de los procesos de formulación de política exterior sin dejar de lado su posición de informantes al resto de la sociedad civil. Esta posición tiene el riesgo de una excesiva politización de la profesión de los periodistas, ya que al contar con las herramientas necesarias para realizar análisis políticos y tomar decisiones expertas sobre el tema, dejan de lado su neutralidad y se convierten en agentes individuales políticos, que tiene su propio peso en la toma de decisiones así como una legítima investidura de representante de la sociedad civil ( in el paso intermedio del voto o elección).

1 Artículo "Los medios de Comunicación y la Política Exterior" pag 29 a 32 en la compilación de Simón Serfaty: "Medios de Comunicación Masivos y Política Exterior".

2 Simon Serfaty (comp.) Media and Foreign Policy. Ediciones Devenir, Argentina, 1995. Conclusiones de "Medios de Comunicación Masivos y Política Exterior". pag. 342 a 345 


\section{Situación de los medios en Ecuador en 1992}

La información confiable más cercana es la que se encuentra en una publicación del estado de los medios de CIESPAL de 1992. Treinta y cuatro diarios eran de propiedad privada mientras que solo uno era de la Iglesia y uno de socios. Esto nos indica que los medios expresan opiniones que son controladas por un solo dueño con intereses personales muy claros y con tendencias identificadas; las noticias que muestren serán reguladas por sus líneas editoriales, así como también influye el factor dado por la publicidad. Así mismo, algunos nuevos diarios que han aparecido pertenecen a un mismo grupo editorial. Por ejemplo, El Comercio (matutino) y Ultimas Noticias (vespertino) que trabajan en conjunto y tienen diferentes tipos de lectores. En cuanto a la cobertura de los diarios, solamente siete tenían alcance nacional mientras que quince a nivel regional y solo cuatro de cobertura de una provincia. Es interesante mencionar que de estos siete diarios de alcance nacional, cuatro de ellos son producidos en Guayaquil (El Telégrafo - que ahora es propiedad del Estado-, El Universo, Extra y Expreso) mientras que solo dos en Quito (El Comercio y Diario Hoy) y uno en Cuenca (El Mercurio); dentro de estudios académicos y círculos periodísticos se toman como referencia de diarios con información "seria" a $E l$ Universo, El Comercio, Diario Hoy y con menores referencias a El Mercurio, más por su circulación que por su contenido. Es también conocido que por tiraje Diario Extra es uno de los más grandes y leídos en el país (especialmente por estratos medios y bajos), pero es descartado por su información sensacionalista y crónica roja cruda. De los cinco de circulación regional, todos pertenecen a la Costa y ninguno a la Amazonia o Galápagos.

Por su año de fundación, entre los diarios más importantes, El Telégrafo es el diario más antiguo del país, creado en 1886, seguido de El Comercio en 1906 y El Universo en 1921. Aproximadamente la mitad de los diarios más conocidos fueron creados antes de los años treinta o después de los sesenta existiendo una gran brecha con la creación de pocos diarios en estos años. El boom de creación de nuevos diarios fue en la década de los ochenta con la aparición de quince diarios de cobertura regional o local. Se manifiesta la tradicionalidad que marcaron por décadas los diarios más importantes y que ha tenido un legado hasta nuestros días. Recientemente se ha visto un florecimiento de nuevos periódicos que han cambiado este panorama y que permiten una mayor elección de los 
lectores; el aparecimiento de revistas especializadas compite con los periódicos por su contenido atrayente al lector que busca información específica.

En cuanto al número de páginas que es proporcional a la información que se transmite (y también a la publicidad que se emite), los diarios nacionales son los que mayores páginas ocupan, entre 22 y 40, con excepción de El Universo que editaba más de 42 y Expreso con menos de veinte. Esto significa que los diarios nacionales están de acuerdo a su distribución de público lector, ya que cubren mayor cantidad de información y pueden mostrar de mejor manera lo que sucede en la realidad cotidiana de su lugar de edición así como de todo el país y el mundo. Eso es claro y muy común que los diarios dependan del lugar de edición para dar mayor importancia a lo que suceda en su ciudad de origen con mayor detalle. Por ejemplo, El Comercio tiene varias páginas dedicadas solo a lo que sucede en Quito y, de la misma manera, El Universo para hechos que suceden en Guayaquil.

La mayoría del resto de diarios en el país manejan ejemplares que tienen entre 12 y 30 páginas que están de acuerdo a su tiraje regional y a la información más limitada que ofrecen a sus lectores, así como una mayor especialización en asuntos locales. En cuanto al alcance por número de lectores, CIESPAL admite que no es una fuente muy confiable pero aferrándose a estos datos, $E l$ Universo era el mayor diario con más de 160 mil ejemplares diarios seguido por El Comercio y Diario Hoy, que tenían entre 30 mil y 100 mil. Los periódicos locales o regionales tenían tirajes bastante limitados, la gran mayoría (80,5\%) no supera los 30 mil ejemplares por día y la mitad de ellos ni siquiera supera los 5 mil por día.

En cuanto al tratamiento de la información, que se da dentro de los diarios, se la agrupan por ejes temáticos pero dentro de un solo bloque en su mayoría (casi 70\%) mientras que solo los diarios nacionales los agrupan en dos o tres bloques. Además, las revistas especializadas no eran muy comunes para esas épocas, mientras que hoy se han diversificado más las tendencias y se han vuelto más comunes las revistas especializadas, según el día, para niños, jóvenes, temas familiares, deportivos o económicos.

Una relación entre el papel de la publicidad dentro de las páginas de los diarios es muy necesaria de mencionar. Los diarios con cobertura nacional imprimen el $48 \%$ de sus páginas con publicidad, esto se debe al mayor número de alcance por tiraje, que los vuelve más atractivos para las empresas que quieren llegar a un mayor consumidor potencial; otra causa es una alianza con grupos 
económicos poderosos, que permiten mantener los diarios a flote con publicidad asegurada y la última es que su mayor cantidad de páginas le permiten mayor flexibilidad a la hora de colocar anuncios y noticias sin quitar peso a los hechos ni a las opiniones. En diarios regionales o locales se mantiene una tendencia entre $36 \%$ y $38 \%$ destinado a publicidad debido a su menor alcance de público y menor espacio en las páginas.

En cuanto a la distribución del espacio de noticias, el análisis contempla todos los diarios del país, por lo tanto es predecible que la mayoría de noticias tendrían un tinte local (62\% del espacio), debido a la gran cantidad de periódicos locales o regionales que obedecen a informar sobre hechos que suceden dentro de sus límites. En los diarios regionales la prioridad la tienen los deportes y los avisos clasificados (también como fuente de ingresos); además los diarios de carácter sensacionalista están ubicados en esta categoría también por su carácter local y de confianza para mostrar su contenido. En los diarios nacionales, el primer lugar es ocupado por los deportes nuevamente, y aquí ya aparece la política internacional con un tratamiento porcentual igual que la política interna. El siguiente tema en la escala es el tratamiento para Cultura y Educación, que también se considera importante por la necesidad de los diarios de masificar cultura y tener alcance nacional para transmitir una cultura de carácter nacional. Entre los tres tipos de diarios se menciona que, porcentualmente, se deja entre $8 \%$ y $10 \%$ para artículos de opinión, editoriales y comentarios; esto significa que dan bastante apertura a los articulistas: así es como quieren expresar sus opiniones como diario de manera formal y segura.

Sobre las fuentes de información, los primeros tres lugares de agencias que proveen de noticias son AFP, EFE y REUTERS. Las tres agencias son de nivel internacional con sedes en Europa o EEUU, con lo cual las cadenas latinoamericanas o nacionales quedan relegadas. Las agencias poseen corresponsales en varias partes del mundo, con lo cual la información que transmiten es importante para las secciones de corte internacional en el ámbito que fuera, como deportes, farándula, política o ciencia. Con la creación de alianzas de tipo ideológico en el continente, han surgido modelos de transmisión de datos regionales como TeleSur, que han mejorado la capacidad operativa para transmitir información a nivel internacional sin depender de otras agencias de noticias tradicionales.

Dentro del mismo tema de transmisión de información es también interesante resaltar que 26 periódicos afirman mantener corresponsales dentro del país, para poder informar directamente sobre la situación nacional. Es claro que 
no solo los periódicos de tiraje nacional se preocupaban de proveerse de la mayor cantidad de eventos en el país, sino también los de alcance regional o local procuraban tener información de primera mano, especialmente en los centros de noticias como son las ciudades principales. En el ámbito internacional, en cambio, solamente dos diarios (Expreso y El Mercurio) poseían corresponsales permanentes en el exterior, no se especifica el lugar, pero ello permite dar cuenta de que la información que estos corresponsales enviaban llegaba directamente al diario para su posterior tratamiento interno; en cambio, para los demás diarios debían esperar la circulación internacional de la noticia de la agencia correspondiente para poder acceder a la misma información.

Durante el conflicto con Perú, y en varias etapas de la negociación, varios medios de comunicación, entre ellos algunos diarios, enviaron corresponsales a Lima para una mejor cobertura de las noticias desde la capital peruana y así mismo de los medios peruanos para transmitir declaraciones, acciones y reacciones de la prensa peruana sobre los hechos que sucedían en la frontera.

\section{Análisis de medios en el conflicto Ecuador - Perú}

El rol de los medios de comunicación en este conflicto es muy interesante, pues el análisis enfoca las posturas de medios escritos expresadas en sus editoriales. El hecho de centrarse en los editoriales se debe a que estos son las posturas oficiales del informativo, y con ello se puede tener una idea más precisa sobre el tratamiento de esta noticia; ya sea la importancia que ha cobrado y la posición del diario con respecto a lo que haya pasado. También se puede identificar la postura hacia el tratamiento y edición de las noticias, en este caso el espacio y modo de ubicar a los hechos relacionados con la resolución del conflicto y sus actores. Los medios, al ser capaces de manifestar su opinión al resto de la sociedad, la pueden influir en cierta medida. Pueden contagiar su visión de la realidad al resto de la población y conseguir apoyo a ciertos planteamientos.

Durante un proceso de negociaciones de paz, el papel que cumplen los medios es mantener la atención en un alto o bajo nivel para la audiencia; la profundidad de su cobertura, las opiniones de respaldo o rechazo a los acuerdos alcanzados y el espacio dedicado al proceso son claves para masificar este hecho bajo su perspectiva. En el caso que analizamos en este trabajo, “...la política exterior ecuatoriana no ha podido desarrollar una agenda de intereses concretos 
a mediano y largo plazo"; 3 por lo tanto, el rol de los medios es difícilmente cuantificable, ya que no puede ser contrastado con proposiciones claras, que ha manifestado el Servicio Exterior. Es entonces clave el contrastar las acciones que el Estado emprendió en este proceso de conflicto y negociación, frente a la postura que los medios escritos mantuvieron y socializaron hacia la ciudadanía.

Gracias a un análisis estadístico previo de la situación en los diarios $\mathrm{El}$ Comercio y El Universo, se conoce que durante 1998, año de firma de la paz, salieron 131 editoriales sobre este tema que expresaban las posturas de los diarios y que fueron moldeando en cierta medida la opinión pública nacional. En esta parte mencionaremos ciertos aspectos considerados durante el conflicto bélico, y que después podremos contrastar con el análisis de las negociaciones. Durante largo tiempo los gobiernos y medios de ambos países se esforzaron por mostrar su versión de la historia, señalar a sus oponentes de una manera degradante y denunciar sus posturas como erróneas y parcializadas. Dentro de este contexto se enraizaron expresiones dialécticas con el enemigo, que buscaban mantener el control social del país así como un aumento de popularidad del gobernante: "Las fervientes expresiones nacionalistas a menudo son el mecanismo de propaganda política interna, para los que aspiran al poder, y asimismo un mecanismo de supervivencia para los que la ostentan". ${ }^{4}$

Poco a poco la atención de los medios se fue centrando en las diferencias entre los dos pueblos así como la gran enemistad producto de los cambios en la delimitación territorial a través de la historia de las dos naciones. Cuando llega el punto de la guerra de 1995, los medios tenían un discurso muy similar al que manejaba el Gobierno y por ello su gran afinidad en la transmisión de la información. Hasta la fecha del conflicto la versión ecuatoriana siempre se basó en razones jurídicas: análisis idealistas de apego a la ley y los tratados suscritos así como el rechazo al uso de la fuerza y a la amenaza o chantaje en discusiones diplomáticas. Una real política exterior en este tema se desarrolla a partir de este conflicto; se deja de considerar primordial el peso jurídico y se procede a

3 Jimmy López (2004). Ecuador-Perú. Antagonismo, negociación e intereses nacionales. Quito: Ediciones Abya-Yala-FLACSO, Pág. 49.

4 José Antonio Carranza en "Relaciones Bilaterales Ecuador y Perú". Artículo dentro de Adrian Bonilla (2002) Orfeo en el infierno, una agenda de política exterior ecuatoriana. Quito: FLACSO-CAFAcademia Diplomática..Pág. 143. 
tomar en cuenta factores de equilibrio de poder, de negociación y mediación y de reconocimiento de interdependencia en varios aspectos.

El equilibrio de poder se maneja dentro de las relaciones entre dos países; cuando se dio el caso de Ecuador, que consideró ganar la guerra del Cenepa, los medios se dedicaron a cuidar esa imagen de victoria, de capacidad real de poder, poner condiciones que históricamente Ecuador se vio limitado para lograrlo. La postura de María Cristina Mata ayuda a entender el papel de los medios, pues afirma que estos cumplieron su deber de informar sobre "la seguridad de las naciones o bandos en pugna y la seguridad para la consecución de la victoria". ${ }^{5}$ Dentro de esta visión, el papel de los medios escritos de cada lado debió ser el de velar por sus intereses nacionales, así como asegurar cumplir los objetivos que se propusieron en las negociaciones. El interés nacional de Ecuador se demostraba en la invalidez del Protocolo de Rio de Janeiro y de Perú en hacer cumplir compromisos ya adquiridos: "por el lado ecuatoriano era la aspiración de acceder al Río Amazonas y por el lado peruano era la ejecución y afianzamiento del Protocolo de Río de Janeiro". ${ }^{6}$

En el caso peruano, se menciona que su posición hermética no permitía un acceso a los movimientos de tropas, a declaraciones de explicación sobre los objetivos a conseguir ni a confirmación de noticias. En cambio, por el lado ecuatoriano se permitió una mayor movilidad a los periodistas, un acceso a las zonas en conflicto y una permanente declaración sobre la postura diplomática y militar oficial. Las negociaciones y mediaciones entonces se basaron en el cambio de posturas históricas y en la aceptación de un posible arreglo entre las partes. Debido a que el equilibrio de poder había cambiado y la interdependencia entre los dos Estados era visible, se pudo proceder a una negociación que llevó a la firma de la paz final.

Una vez reconocidos los cambios de postura que fueron necesarios para poder llegar a un proceso de paz, es importante considerar la cobertura que los medios otorgan en sus espacios de noticia o de opinión como un hecho fundamental para transmitir la postura hacia el resto de los ciudadanos; el hecho de

\footnotetext{
$5 \quad$ Véase su artículo "Medios Masivos y Conflicto, existe una sola lógica", publicado por la FLACSO durante el proceso de negociación de la paz, para darse una idea sobre las versiones de los dos lados del conflicto y analizar ciertos aspectos sociales que intervienen en el proceso.

6 Jimmy López. Ecuador-Perú. Antagonismo, negociación e intereses nacionales. Ediciones Abyayala-FLACSO, Quito, Ecuador. 2004. p. 52.
} 
ganar la batalla o de lograr una victoria diplomática no significa nada si no se logra transmitir esta información a la audiencia; "la guerra no es una sino tres, afirma un adagio sajón que alude a las batallas en las trincheras, las batallas diplomáticas y las batallas informativas". ${ }^{7}$ La batalla militar es netamente de soldados que sacrifican sus vidas en defensa de lo que creen es valioso para su país y lo que es ordenado por sus superiores; el diplomático lucha por hacer prevalecer lo ganado en el territorio de batalla o también por sacar ventaja de los vacíos en las negociaciones; finalmente el periodista informa sobre lo que sucede y transmite lo que sabe hacia el resto de la sociedad. Este papel de transmisor le permite recoger su versión del campo de batalla y de la mesa negociadora, para transmitir aires de triunfo a sus conciudadanos o para presionar más a los tomadores de decisiones.

Es así que la batalla informativa de ciertos medios ecuatorianos durante el conflicto fue el de mostrar la cara fea de los enfrentamientos y crear un ambiente negativo frente a la continuación del conflicto frente a los beneficios de la paz. Al crear este ambiente pro paz, los artículos informativos y de opinión se enfocaron en resaltar los recursos económicos utilizados en la guerra, y que podrían haber sido utilizados en proyectos de desarrollo comunitarios, en el caos familiar de los caídos en combate, en el fuerte vinculo entre las comunidades fronterizas; en fin mostraron el conflicto como el "destructor de la posibilidad de desarrollo de los pueblos de ambos países". ${ }^{8}$ Esta creación de una opinión pública favorable a las negociaciones de paz dio pie a un ambiente, que pedía la paz a cambio de mejores beneficios, que a la larga se propusieron en los acuerdos binacionales de cooperación. También permitieron un mayor acercamiento entre comunidades fronterizas por medio de proyectos conjuntos, que facilitaban el contacto intercomunitario y favorecían el entendimiento mutuo.

En el caso del conflicto con Perú, la necesidad de una apertura militar a la información del conflicto fue clave en Cenepa. Ecuador aprendió esa lección con el conflicto de Paquisha, donde el hermetismo y la incapacidad de transmitir su versión de los hechos dieron una gran ventaja al Perú, al permitirle mostrar la versión peruana al mundo. En el conflicto del 1995, en cambio, Ecuador

\footnotetext{
$7 \quad$ Adrian Bonilla (1999). Ecuador-Perú. Horizontes de la negociación y el conflicto. Quito: FLACSO. p. 249.

8 Ibíd., p. 250.
} 
tomó la iniciativa y dejó que la credibilidad de sus afirmaciones sea la regla del trato con los medios así como la mayor apertura a los medios para coberturas en el lugar de conflicto y en bases ecuatorianas. La cobertura de las posiciones en disputa fue clave, y el rol que los medios ecuatorianos tuvieron fue importante al mostrar a la comunidad nacional e internacional los destacamentos como Tiwintza, Cueva de los Tayos y Base Sur en posesión efectiva de militares ecuatorianos. Construir lo que denominan "países hermanos" no se logra solamente mediante la rúbrica de presidentes en un Protocolo, ni revisando libros de historia; se logra construyendo entre los diferentes actores de dos países objetivos comunes, necesidades reales y estrecha fraternidad y solidaridad.

\section{Diplomacia de alto nivel}

En el año 1998 se vivió la última etapa de las negociaciones de paz, y durante el mes de octubre ya se vieron estancadas las negociaciones por una negativa de Ecuador de dar paso a ciertas propuestas en el marco de la delimitación de la frontera. La solución encontrada para superar este problema fue el uso de la "diplomacia de alto nivel" con el diálogo directo de los presidentes de ambas naciones. Durante este tiempo de negociaciones directas, los medios se encargaron de reproducir información sobre cómo se realizaba el proceso y pusieron bastante énfasis en los logros de una paz beneficiosa para los dos países. Desde comienzo de mes se resaltaban los desafíos que el Gobierno de Mahuad (recién posesionado a esa época) debía afrontar, entre ellos el rol de negociador final de la propuesta de paz con el Perú. Las negociaciones previas ya habían sentado acuerdos sobre la mayoría de temas como Desarrollo Binacional, Medidas de Confianza, Soberanía fluvial en el Amazonas y el Canal de Zarumilla. Sin embargo, todavía faltaba la delimitación final de la frontera. El rol de los garantes es tomado muy en cuenta por los medios, los mencionan varias veces en el transcurso de sus editoriales para dar legitimidad al proceso; el papel de Argentina, Chile y principalmente Estados Unidos y Brasil fue visto con buenos ojos por la prensa, para llegar a la que se denominaba la solución final.

Además El Universo en su editorial del 11 de octubre menciona la importancia del rol de los garantes, para poder equilibrar la "estatura física" de los dos países en términos internacionales. Siguieron de cerca la manera en que los países garantes fueron introduciendo sus posturas en las conversaciones; en primer lugar manejando un papel secundario, de mediadores, para poste- 
riormente llegar a ser los tomadores de decisiones finales dentro del proceso de paz de Ecuador y Perú. Llegaron a decidir acuerdos en base a parámetros enfocados en acuerdos jurídicos previos; su papel activo en el último tramo de la negociación fue clave para acelerar el proceso, y así dieron cuenta los diarios con sus llamamientos a la justicia e imparcialidad de los garantes. El Comercio menciona, en su editorial del 9 de octubre, el afán de los mandatarios de "entrar en otra etapa de la historia ecuatoriano-peruana, saliendo de una situación obsoleta y difícil".

El papel de personalidades específicas en el proceso de relaciones exteriores es la clave, que se manejaba a nivel teórico desde un comienzo de esta investigación. Los medios informan que el rol de los presidentes Enrique Cardoso y Bill Clinton fue un aliciente para poder llegar a un buen término, mediante la visita a Estados Unidos y la Conferencia Iberoamericana en Oporto se refleja un ambiente oportuno para la intervención de estos individuos y de sus delegados: la prensa ratifica esta posición de aceptación a su papel mediador, para lograr una mejor coordinación en las etapas finales de la negociación. El pedido que realizaron las partes para una mediación, que posteriormente se volvió vinculante en su decisión, les permitió una mayor maniobra a los garantes para enrumbar el proceso de paz hacia la firma final. Los medios contribuyen, al resaltar el involucramiento histórico de estos países, así como la necesidad de contar con un respaldo internacional; el pronunciamiento de los diarios a favor de esta propuesta, así como el llamamiento a las partes para que acepten el pedido de los garantes, es una muestra que apoyaban un proceso de paz aun con resultados inciertos, debido al alto costo que significa mantener una guerra y un estado latente de conflictividad.

Mientras El Universo enfatiza la efectividad de una fórmula presentada por los garantes, en la confianza en que la comunidad internacional apoye esta propuesta y en la postura ideal del Congreso Nacional dejando de lado partidismos, en El Comercio predomina el llamado a dejar planteamientos políticos disidentes al margen, y concentrarse en lo que sería una paz con todas sus consecuencias en niveles económicos, sociales y de régimen internacional. Se mencionan también los peligros de entregar "un cheque en blanco" a los garantes, para lograr la paz, pero afirman que el riesgo es común a los dos países; mientras Perú estaba sacudido por un clima electoral nacional, Ecuador presentaba problemas económicos, por lo tanto la opción de otorgar por medio del Congreso a los Garantes la facultad de decidir por una vez sobre el destino de la Paz era 
una acción arriesgada, pero que limitaba los márgenes de maniobra de los dos mandatarios y no comprometía concesiones de ningún lado. El Universo se refiere a la confianza en que no exista "un acuerdo previamente pactado" en su editorial del 16 de Octubre, mientras que El Comercio menciona, el 11 de octubre, que se necesita llegar a "una fórmula que permita un acuerdo global y definitivo sobre el diferendo limítrofe".

\section{Actores secundarios}

Es interesante también ver el rol de ciertos actores secundarios que intervienen en el proceso de las negociaciones y que son mencionados por los medios. El mayor ejemplo es la figura del Papa Juan Pablo II: cuando el pedido de los garantes fue contestado, influyó el aval de Su Santidad en la firma del convenio que resultare de esto; y efectivamente, así sucedió. Durante la firma de paz en Brasil, un enviado especial del Vaticano estuvo presente para dar la bendición a un documento de hermandad entre pueblos. Esta figura no sería muy importante, si no fuera por las tradiciones católicas de los países y de sus sociedades, la facultad del Papa de poder intervenir y dar aval en un asunto de relaciones internacionales tiene cabida en tanto su papel religioso representa una institución con un alto prestigio transversal a toda clase dentro de la sociedad.

Otro aspecto interesante a tomar en cuenta es la importancia de la sociedad civil; los medios se autoproclaman como los portadores de esta voz y en nombre de ella emiten criterios. Un ejemplo claro es el editorial de El Comercio del 14 de octubre, donde se menciona que la sociedad civil ha venido siguiendo el proceso de cerca, celebrando los avances y lamentándose de los contratiempos, y al mismo tiempo menciona que la sociedad está presta y con buena voluntad hacia la consecución de una paz constructiva. Es un llamamiento a los tomadores de decisiones para que agiliten el proceso y se pueda ver un final pronto, aunque la sociedad no les ha dado ningún permiso para que se hable en su nombre, la prensa en base a encuestas se atribuye ese deber.

Es claro que los medios tienen la capacidad de hacerlo, son los representantes más notorios e influyentes de una sociedad muda, la autodenominación de los medios como su representante se lo toman muy en serio y se refleja en la atribución de criterios, pedidos y llamamientos en nombre de toda una sociedad. El 11 de octubre, El Comercio menciona que según las encuestas, la mayoría de ecuatorianos y peruanos están en busca de la paz. Posteriormente, 
El Universo muestra, el 25 de octubre, los resultados de una encuesta realizada por CEDATOS sobre el apoyo a la firma de paz con el Perú y el problema limítrofe: se muestra que el $48 \%$ aprueba de manera general el tratado frente al 39\% que desaprueba, el $47 \%$ está de acuerdo con la propuesta de los garantes frente al $31 \%$ en desacuerdo; casi 7 de cada 10 ecuatorianos quisieran que la zona de conflicto se transforme en zona de conservación ecológica y existe un ligero desacuerdo ( $46 \%$ en contra frente a $40 \%$ a favor) de la propuesta de Tiwintza como propiedad ecuatoriana en soberanía peruana.

\section{Continuidad en procesos de cambio}

Una postura interesante en cuanto a continuidad del proceso se puede revisar en El Universo, cuyos lineamientos editoriales manifiestan la necesidad de procesos continuos, de adhesión a instrumentos jurídicos y herramientas internacionales para la resolución de conflictos. Cuando en los comienzos de octubre se producen unos cambios en el Gabinete peruano por diferencias sobre el estado de las negociaciones con Ecuador, este diario hace un llamamiento a continuar con el proceso aún con discrepancias de los tomadores de decisiones, ya que esto permite ser consecuentes con Políticas de Estado ya determinadas y firmes.

El interés de la prensa por mantener funcionando la representatividad del Estado como tomador de decisiones es muy interesante; no deja de lado la importancia que los individuos tienen en el proceso de negociación, pero al mismo tiempo ratifica la necesidad de contar con objetivos claros que deben ser seguidos a toda costa para conseguir beneficios mutuos. Así mismo permite con sus pronunciamientos el rescate de la institucionalidad a nivel internacional representada en la validez del Protocolo de Rio de Janeiro y en la Declaración de Santiago: en base a estos instrumentos jurídicos se produjo el pedido a los países garantes para dar una solución definitiva al proceso negociador. Se rescató el valor jurídico de los cuatro países garantes, aún cuando se cuestionó la manera en que históricamente formaron parte del Protocolo de Rio de Janeiro denunciado por Ecuador.

El escenario internacional toma bastante fuerza al coincidir el tiempo de las negociaciones con la Cumbre Iberoamericana. Esta cumbre tenía como uno de sus objetivos a tratar los ejes de la globalización e integración. El rol que cumplieron los países garantes en este foro multilateral se conectó con las acciones 
de los países en negociación y a sus Congresos. En estas fechas se aceptó la propuesta vinculatoria de los garantes para definir los términos en los que se firmaría la paz, se vinculó este tema con la integración sudamericana y con la multilateralización de la resolución de conflictos. Se resalta la importancia para la región de saldar uno de los conflictos más largo del continente, en un intento por convertir a la región en una isla de paz, atractiva para las inversiones y para el turismo.

Asimismo la internacionalización de la solución del conflicto pasa por la aceptación de otras naciones del proceso y con ello el reconocimiento de validez para que sea un acuerdo duradero y vigilado por otros actores fuera de los involucrados históricamente. El compromiso de los dos países en acatar la decisión final para dar fin a un problema largo y doloroso es aceptado y aplaudido por los países iberoamericanos. La confianza en que la propuesta contenga principios de equidad y validez judicial merece análisis extensos en la prensa de ambos lados. Masifican la sensación de aproximarse a la tan anhelada paz entre los dos pueblos, y sus mensajes se enfocan en exaltar la grandeza de este paso para las dos sociedades.

\section{Conclusiones}

La aproximación teórica nos permite dar cuenta de una relación muy íntima de los medios de comunicación con la sociedad y, por ende, con las decisiones políticas; la posición manejada comúnmente habla de una separación entre los medios y las decisiones políticas, ya que a simple vista no parece haber una conexión directa entre los dos campos, pero el momento de entrelazar los medios de comunicación como masificadores de propuestas mediante teorías como la espiral del silencio o la sociedad persuasora se encuentran bastante indicios de su grado de influencia en temas políticos, económicos y culturales.

La sociedad funciona como un todo y a la vez por medio de ciudadanos individuales que permiten la heterogeneidad. Cuando están en juego posturas políticas, los cambios que pueden producir los medios al decidir publicar ciertas noticias y al dar mayor o menor importancia a las mismas es clave en la forma cómo los individuos percibirán el entorno en que se desenvuelven. Una persona solamente puede tener un conocimiento fáctico y directo de lo que sucede a su alrededor y de lo que realiza en su vida cotidiana. En adición a esto, por medio de la prensa, radio o televisión, el ciudadano puede descubrir un mundo ajeno 
a su capacidad real de sensación pero al mismo tiempo puede ser susceptible de afectarle. Es en este punto cuando los medios toman este rol de formadores de opiniones, de realidades y de imaginarios, logrando atravesar radicalmente todo aspecto de la vida del ser humano y permitiendo una visión más amplia de la realidad.

Las nuevas teorías mencionan la importancia de considerar la existencia de una sociedad trasnacional con individuos que trascienden las fronteras físicas de los Estados-Nación. La posición de Castells menciona la importancia de la tecnología en la vida diaria que es fundamental para poder entender los procesos de transmisión de la información, por medio de la velocidad de transferencia, de la reducción de interferencia y de la precisión de la capacidad receptora; estas facilidades actuales permiten a un ser humano conocer sobre la realidad que los medios de comunicación le muestran del mundo así como sobre su misma localidad. La idea de globalidad encaja perfectamente con esta apreciación.

Claro está que las dificultades de acceder a este nivel de comunicación radican en los servicios de cobertura, que un Estado pueda ofrecer en sus fronteras nacionales, la educación tecnológica de los individuos y la capacidad de reacción en tiempo real entre emisor y receptor del mensaje. La capacidad de los medios de transmitir expresividad, rapidez y difusión les permite alcanzar un mayor publico cautivo; el hecho que las noticias puedan llegar de manera efectiva puede catapultar acciones a favor o en contra de los eventos pasados y mientras mayor sea el público al que llega la noticia, mayor la reacción por medio de ideas, sentimientos o acciones.

El momento en que estas ideas globales ya traspasan las fronteras nacionales, entonces los medios adquieren una importancia universal también. Su rol atraviesa los diferentes aspectos de la vida cotidiana y le permiten trascender también sobre asuntos internacionales. El modelo realista basado en la importancia del Estado-nación pierde su importancia dando paso a la teoría de la transnacionalidad en diversos aspectos, la pérdida de capacidad de acción del Estado en temas emergentes o en temas que requieren la acción de varios actores internacionales dan lugar al aparecimiento de nuevas tendencias, nuevos actores y nuevas relaciones de poder.

Las teorías de relaciones internacionales debaten arduamente sobre el papel que deben tener los Estados dentro de esta nueva organización global, así como el nivel de interacción que debe tener la sociedad, por ejemplo, en temas como movilidad humana, de capital y de recursos son ampliamente discutidos 
por académicos y políticos para tomar cursos de acción. Aunque la toma de decisiones sigue en mano de los representantes oficiales del Estado, los tramos de análisis y planificación son apoyados, discutidos y moldeados por actores estatales, no gubernamentales e internacionales.

En el caso especifico de los medios de comunicación existen organizaciones que agrupan a diferentes diarios a nivel regional o global así como a las agencias emisoras de noticias; el rol de los medios de comunicación no es todavía suficiente para poder hablar de regímenes internacionales de los medios pero si es lo suficientemente poderoso como para influir en otros actores de la arena internacional. Esta capacidad de influencia viene dada por su misma característica de representante de la sociedad civil así como creador de propuestas y defensor de la libertad de expresión. Aun cuando sus planteamientos responden a sus grupos editoriales se consideran una fuerza diferente del poder político tradicional; en las democracias occidentales son un pilar para mantener un Estado de Derecho y precautelar las libertades fundamentales como lo demuestran las continuas medidas para calificar a una democracia según los accesos y libertades de los medios.

Cuando se logra analizar de manera profunda la realidad teórica y la manera como esta nos permite comprender la situación actual, el conflicto de Ecuador con Perú es el referente más próximo que se tiene con la suficiente distancia histórica e información para poder analizar de una manera apropiada. Retomando afirmaciones teóricas de Livingston, el conflicto de Ecuador-Perú se encaja dentro de la categoría de un conflicto de disuasión táctica al ser una respuesta rápida en un lugar específico y mantener el interés de los medios muy alto al momento del conflicto con un gran despliegue de periodistas que actuaron como multiplicadores de información en todo el mundo dando a conocer posturas de un bando al otro. Cuando el conflicto se calmó y vino el proceso de alto al fuego y la negociación de paz, se encaja en la categoría "Procesos de Paz" y "Mantenimiento de Paz" que se caracterizan por un uso restringido de la fuerza con atención mediática en esos eventos, la creación de contingentes militares de control y con procesos largos de negociación donde la atención de los medios se concentra en las partes finales de los acuerdos o en posibles escaladas de violencia.

El tema de los medios de comunicación no cobró demasiada atención cuando se realizaron los análisis coyunturales en la época de conflicto y de negociación; mas ahora, transcurrido algún tiempo, empiezan a surgir las aproxima- 
ciones teóricas tendientes a entender el papel de ciertos actores en el proceso. Con las nuevas visiones del mundo como un conjunto de sistemas los medios adquieren una mayor importancia en las relaciones internacionales; en nuestro caso específico, América Latina ha empezado un proceso de control o veeduría a los medios de comunicación por medio de leyes estatales y con el rechazo de organismos transnacionales como la SIP. Los regímenes burocráticos de las naciones ven cómo su poder e influencia se ven mermados o criticados por una prensa cada vez más libre y con mayores aspiraciones, por lo tanto se ha tomado el camino del control dentro de las fronteras nacionales sin el apoyo de otros actores internacionales; obviamente, el rechazo que se presenta responde a los intereses propios de quienes conforman los medios de comunicación y en base a fundamentos históricos de las sociedades democráticas occidentales.

Se puede concluir que el papel de los medios fue muy activo durante todo el tiempo desde 1995 a 1998: tuvieron bastante movilidad para declarar sus puntos de vista, para acceder a información de primera mano y para contrastar propuestas oficiales. También se destaca su capacidad de profundización en temas limítrofes con el vecino del sur: de manera especial en asuntos de análisis de las consecuencias y beneficios de la paz y en aproximaciones jurídicas e internacionalistas de posibles soluciones. Finalmente su rol se manifestó fuertemente en la sociedad por ser los portavoces de la unidad nacional (durante el conflicto), de la salida negociada de la paz (durante y después del conflicto) y de los beneficios inéditos que podría acarrear la paz (al final de las negociaciones); su capacidad de masificar estos análisis y propuestas les permitió ser actores clave en la formación de opinión pública y en el cambio de percepción histórica sobre la situación con Perú. Después de firmada la paz, su rol se redujo por la presencia de otros eventos y noticias que coparon la atención mayoritaria, pero no se dejó de lado la cobertura constante de los avances de la ejecución de los acuerdos ni de las dificultades de los procesos de cooperación.

Cuando se analiza el rol de los medios en cuanto a nivel de influencia sobre los tomadores de decisiones, la curva es completamente opuesta, los medios mantuvieron una muy baja influencia durante la época de conflicto, el rol principal lo mantuvieron los militares y los puestos políticos de mayor jerarquía; más bien, una vez empezadas las negociaciones, los medios pudieron influir en el pensamiento de los tomadores de decisiones como en el de toda la sociedad propiciando un ambiente de optimismo y oportunidad en el proceso. Existe una dualidad del efecto de los medios de comunicación en la sociedad: mientras las 
decisiones en política exterior se mantengan en el más alto nivel, la influencia de los medios puede ser menor; en cambio mientras más se desconcentre la toma de decisiones, el rol de los medios es mucho más activo, aunque no de manera directa a los tomadores de decisiones como objetivos sino como resultado de la masiva influencia que proyectan los medios sobre toda la sociedad, al modificar pensamientos, alentar propuestas y fomentar sensaciones.

\section{Bibliografía}

AGUILAR, Ana María

2004 Medios de comunicación y conflictos armados. Tesis. Quito: Pontificia Universidad Católica del Ecuador, Facultad de Comunicación, Lingüística y Literatura.

BALLESTA, Javier (Comp.)

2002 Medios de comunicación para una sociedad global. Murcia-España: Universidad de Murcia.

BONILLA, Adrián (Ed.)

1999 Ecuador-Perú. Horizontes de la negociación y el conflicto. Quito: FLACSO.

2002 Orfeo en el infierno, una agenda de política exterior ecuatoriana. Quito: FLACSO-CAF-Academia Diplomática.

BOTERO, Luis

2006 La generación de opinión pública: ¿Asunto público o privado? Enero. Extraído desde www.saladeprensa.org.

CASTELLS, Manuel.

2002 La Era de la Información: Economía, Sociedad y Cultura, Tomo I: La Sociedad Red. México: Siglo XXI Editores. Cuarta Edición.

2002 La Era de la Información: Economía, Sociedad y Cultura, Tomo II: La Sociedad Red. México: Siglo XXI Editores. Cuarta Edición.

CURRAN, James.

2002 Medios de comunicación y poder en una sociedad democrática. Barcelona: Editorial Hacer.

DIARIO HOY

1995 La Epopeya del Cenepa. Fascículo 5: La Guerra Informativa. Colaboración con la Secretaria Nacional de Comunicaciones. Ecuador. 1995

DONOSO, Claudia

2009 Ecuador-Perú. Evaluación de una década de paz y desarrollo. Quito: FLACSO Ecuador - CAF. 
GUZMÁN, Ana María

2004 La desinformación de los medios de comunicación: golpe de Estado en Venezuela. Tesis. Quito: Pontificia Universidad Católica del Ecuador, Facultad de Comunicación, Lingüística y Literatura

HERRERA, Meliant

2004 "Los Medios de Comunicación Social en la sociedad capitalista actual”, en Revista Electrónica Razón y Palabra. México. http://www.razonypalabra. org.mx/anteriores/n38/mherrera.html.

HUDSON, Valerie

2005 Foreign Policy Analysis: Actor-Specific. Theory and the Ground of International Relations. Phd. Brigham Young University.

LIVINGSTON, Steven

1997 Claryfying the CNN Effect. An examination of media effects according to type of military intervention. Research Paper. The Joan Shorestein Center, Universidad Harvard. Internet: http://www.hks.harvard.edu/presspol/publications/papers/research papers/r18 livingston.pdf.

\section{MENESES, Zuly}

1992 Análisis de Medios de Comunicación en el Ecuador. Quito: CIESPAL.

MINISTERIO DE RELACIONES EXTERIORES DEL ECUADOR

1992 Libro Blanco: Hacia la solución del Problema Territorial con el Perú. Quito.

NAVEH, Channan

2002 "The Role of the Media in Foreign Policy Decision-Making: A Theoretical Framework" en. Revista Conflict \& Communication online, Vol. 1, Núm. 2. Extraído de: www.cco.regener-online.de.

NEUMANN, Elizabeth

1996 La espiral del silencio: Opinión pública: Nuestra piel social. Barcelona: Paidos. Extraído desde www.nombrefalso.com.ar.

NYE, Joseph Jr.

2003 Understanding International Conflicts. An introduction to Theory and History. Ediciones Longman. Cuarta Edición.

ORDOÑEZ, Renán

2004 Análisis crítico estadístico de los editoriales de los diarios El Comercio y El Universo durante 1998, acerca de las negociaciones con el Perú y la firma de paz definitiva. Tesis. Quito: Pontificia Universidad Católica del Ecuador.

ORTIZ Gonzalo

1999 En el Alba del milenio. Globalización y Medios de comunicación en América Latina. UASB-Proyecto Latinoamericano de Medios de Comunicación. Quito: Corporación Editora Nacional. 
PEÑA, Mónica

s.f. "News media and the foreign policy decision-making process, CNN or Washington?", en Revista Electrónica Razón y Palabra. Numero 32. Extraído de http://www.cem.itesm.mx/dacs/publicaciones/logos/anteriores/n32/ mpena.htm.

PLANEX 2020

2006 Relaciones del Ecuador con sus países vecinos (Colombia-Perú). Quito: Ministerio de Relaciones Exteriores.

REVISTA CONFLICT AND COMMUNICATION ONLINE: http://www.cco.regeneronline.de/ (Artículos varios).

RISSE-KAPPEN, Thomas

1999 "Avance en el estudio de las relaciones transnacionales y la política mundial”, en Revista Foro Internacional. Ciudad de México, Octubre-Diciembre.

ROIZ, Miguel

2002 La sociedad persuasora. Madrid: Paidós.

SERFATY, Simón (Comp.)

1995 Media and Foreign Policy. Buenos Aires: Ediciones Devenir.

Envío 23 de enero de 2012 - Fecha de aceptación 2 de marzo de 2012. 